\title{
2006-2314: A NANOTECHNOLOGY EXPERIMENT: DESIGN OF LOW COST SCANNING TUNNELING MICROSCOPES
}

\section{Nebojsa Jaksic, Colorado State University-Pueblo}

Nebojsa Jaksic received Dipl. Ing. degree in electrical engineering from Belgrade University, Belgrade, Yugoslavia in 1984, M.S. in electrical engineering, M.S. in industrial and systems engineering and Ph.D. in industrial and systems engineering from the Ohio State University, Columbus, Ohio in 1988, 1992 and 2000, respectively.

Currently, he is an Associate Professor in the Department of Engineering at Colorado State University - Pueblo. His teaching and research interests include nanotechnology manufacturing and instrumentation. 


\title{
A Nanotechnology Experiment: Design of Low Cost Scanning Tunneling Microscopes
}

\begin{abstract}
This paper describes a set of laboratory exercises where upper-level undergraduate engineering students constructed and tested low-cost scanning tunneling microscopes (STM) of their own designs as an assignment for a nanotechnology course taught during Spring 2005. Before attempting the design task, students were exposed to nanotechnology instrumentation principles, commercial-grade scanning probe microscopes, current design methods and a number of design examples from literature. Three design objectives were met: each instrument cost below $\$ 500$, a sustainable tunneling current was achieved and calculations predicted atomic-resolution capabilities of the designed instruments. While the scans of highly oriented pyrolytic graphite showed surface characteristics the microscopes could not distinguish individual atoms.
\end{abstract}

\section{Introduction}

Nanotechnology is one of the most vibrant research areas today. Some of the research is in the commercialization stage, like carbon nanotube applications in computer monitors or energy storage devices. In academia, at the graduate level, there was a substantial number of course offerings dealing with various nanotechnology topics. There were fewer successful attempts in presenting this material to the undergraduate student population, mostly in sciences, while only a handful of isolated reports documented nanotechnology topics in undergraduate engineering curricula ${ }^{1}$.

During the Spring 2005 semester, a design-based nanotechnology course was offered to upper-level undergraduate industrial engineering students. The course presented an implementation of McCarthy's ${ }^{2}$ version of the $\mathrm{Kolb}^{3}$ learning cycle and was motivated in part by work presented by Harb et $a l^{4}$. A large portion of the course lab segment was dedicated to the design, construction and testing of scanning tunneling microscopes (one of the major instruments in nanotechnology research) of students' own designs. Three engineering criteria were specified: the cost of each STM (not including the PC) below $\$ 500$, the tunneling current duration long enough to perform at least one full scan, and the device capability to achieve atomic resolution.

\section{Previous Work on Low-Cost Scanning Tunneling Microscopes}

Scanning tunneling microscopes present a major tool for nanotechnology imaging and manipulation. Since their invention in 1980 's ${ }^{5}$, they were an important topic of instrumentation research. STM commercialization encouraged their use in many materials laboratories. However, STMs' high cost $(\$ 10,000$ for a student version device or $\$ 50,000$ or more for a research-grade device) limited their use to mainly larger institutions. Even there, undergraduate students are usually not given an opportunity to work with these high-level research instruments. 
There are many books and articles on STM construction ${ }^{6-8}$, but they usually describe STMs that are expensive and do not use off-the-shelf parts. While the academic literature contains some promising information, the Web is often a good choice for exploration of current projects. An extensive Web search revealed three promising projects (either unfinished, not achieving atomic resolution, or using obsolete parts) that were used as a starting point in our preliminary designs. While a successful STM project by Alexander ${ }^{9}$ used very inexpensive components it required two laboratory signal generators and an oscilloscope for operation. It did not have a motorized final approach of the tip towards the sample and the data could not be recorded in a computer file. However, the scanner introduced in this project was studied and implemented. Muller ${ }^{10}$ tried to use a more sophisticated and expensive commercially available STM scanner, a Motorola DSP 56002 digital signal processor (DSP) for digital control and feedback loop, and a PC for data acquisition, display and visualization. Unfortunately, the project was not finished and it used a DSP board that is out of production. An adaptation of the STM project by Alexander', using an independent microprocessor for control and a PC for data acquisition and display was implemented as a senior project at Cornell University ${ }^{11}$. Major weaknesses of this project were the low resolution of the hardware (two 8-bit D/A converters for $\mathrm{x}-\mathrm{y}$ scanning, and one 10-bit A/D converter for tunneling current data acquisition) and only one mode of operation at a constant height that limited its operation to very flat surfaces.

Adali $^{12}$ developed a vibration isolation system and Dobryanskaya ${ }^{13}$ developed a graphical user interface as preparations for student design projects described in this work. The data acquisition and control hardware used was a Texas Instruments' MSC1211EVM board, an external measurement/control module consisting of a multi-channel 24-bit A/D and a 16-bit D/A converter. This board could significantly improve the resolution of STM designs.

\section{Student Design Experience}

Laboratory exercises, experiments and design projects are proven tools used to increase student learning in engineering education. Thus, the design component of this nanotechnology course was emphasized. Students designed, built, and tested their own scanning tunneling microscopes. Some of the lectures and laboratory reports were placed on the $\mathrm{Web}^{14}$.

\subsection{Scanning Tunneling Microscope Lectures}

In lectures, STM concepts and previous designs were introduced. The discussions included the original design 5 , STM theory of operation, tunneling current generation and sustainability, vibration isolation systems, approach mechanisms, scanners, control systems for approach and scanning, modes of operation, data acquisition systems and graphical user interfaces. The work of two MS graduate students was brought to the classroom even before their results were published. A prototype $\mathrm{STM}^{12}$ shown in Figure 1 that was used to test effectiveness of various passive vibration isolation systems was presented. An analysis of unimorph piezoelectric actuators ${ }^{13}$ used in this design was also presented. Since the development of the software user interface would have required 
more than a semester to complete, a minimal graphical user interface was developed and made available for this project.

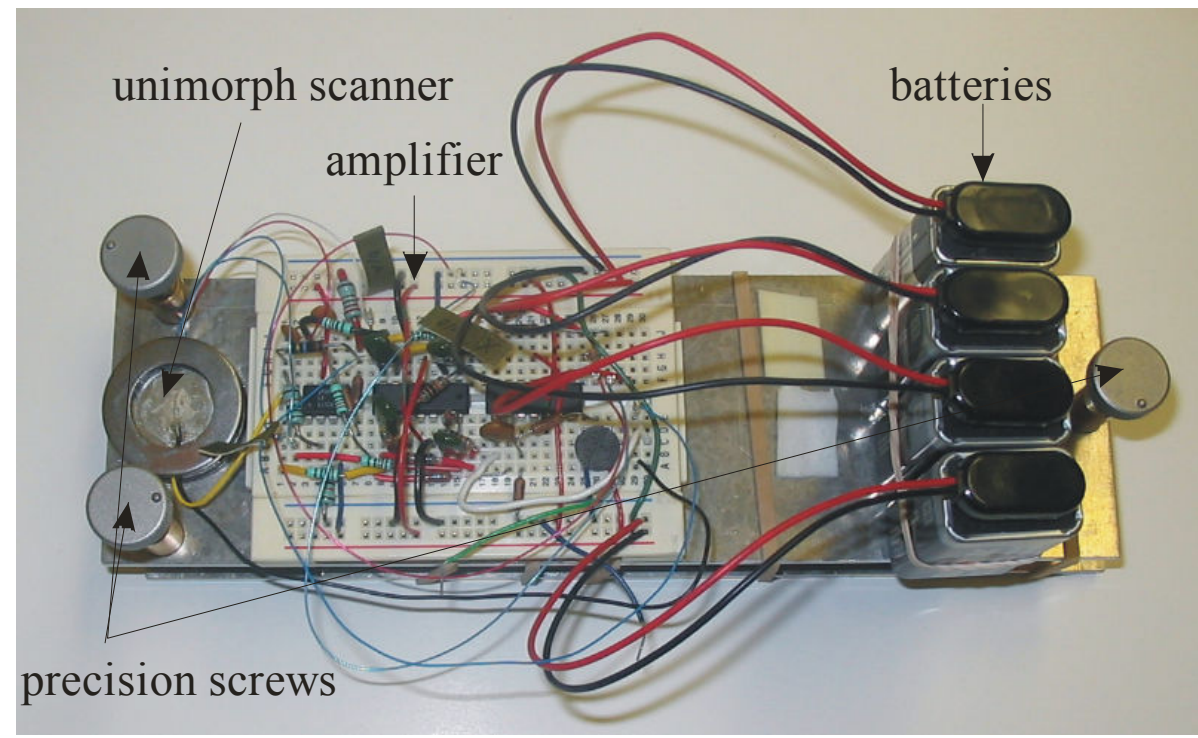

Figure 1. A prototype STM, top view

\subsection{Laboratory Sessions}

The laboratory portion of the course was set up to mimic a typical research environment. Laboratory space was provided for students outside normal lab hours. The machine shop and the shop supervisor were available to help manufacture student designed STM components. The laboratory sessions were coordinated with six hours of lecture time dealing with nanotechnology instrumentation. Two graduate students (one for the electronics and one for the software) were assigned to the project as experts.

The STM design module consisted of five laboratory sessions. Each session was completed in two laboratory hours, except for the construction session that required a number of hours in the machine shop. In addition, students spent a considerable amount of time outside the lab designing their STMs.

\subsubsection{STM Design Principles with Examples}

This first laboratory session discussed many ideas previously used in STM designs. An STM consists of an STM body, vibration isolation system, approach mechanism, scanning device, current amplifier and a controller. A block diagram of a typical STM is depicted in Figure 2. 


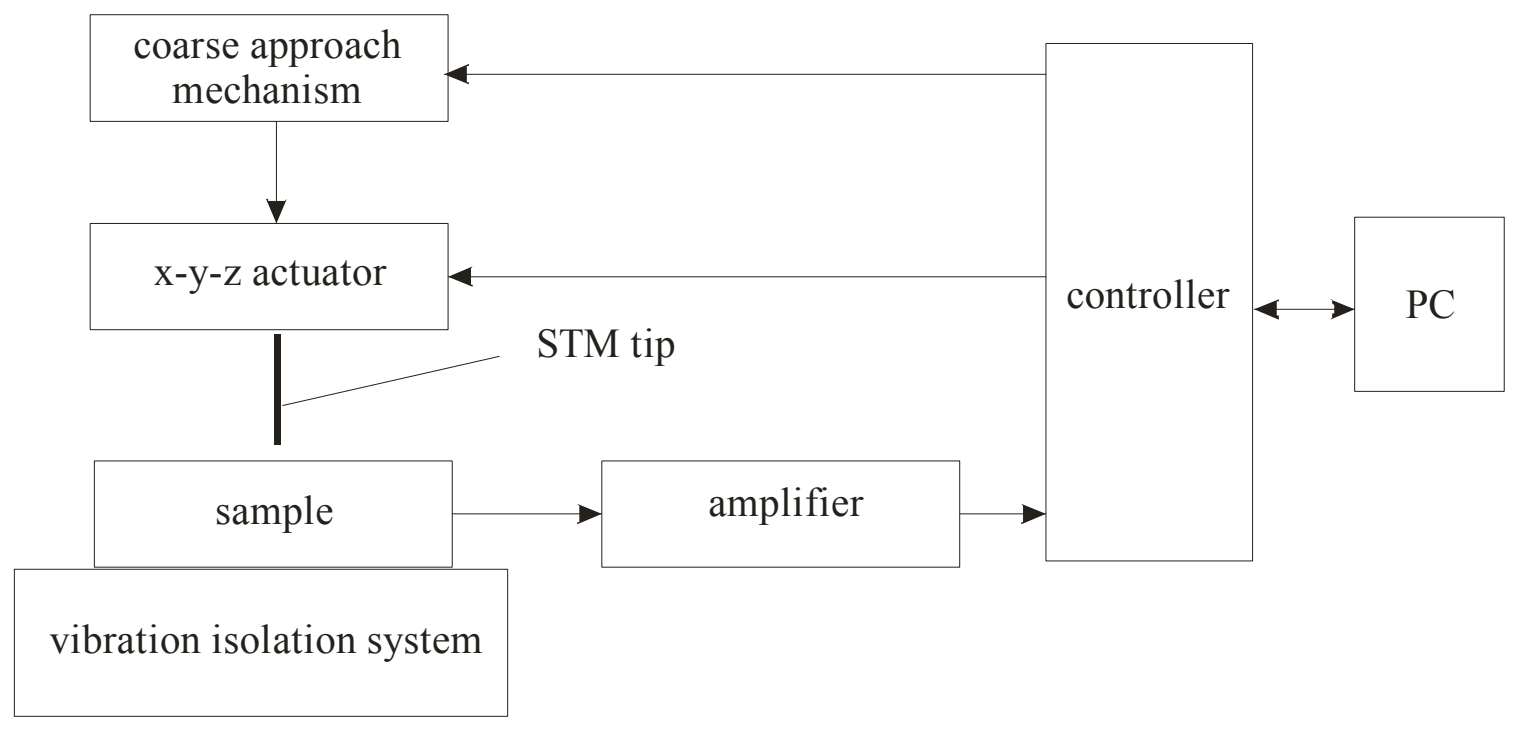

Figure 2. A block diagram of a typical STM

An approach mechanism brings the STM scanner tip within range of the sample to be imaged. Since the tip must be within a few nanometers from the sample for a successful scan, the mechanical attenuation of the approach mechanism must be high. In addition, the distance between the scanner tip and the sample must be reasonably constant for the duration of the scan. If the tip moves too far away from the sample, the tunneling current indicating the height of the scanned point may be lost. If the tip of the scanner moves too close to the sample it may crash into it. The crash of an atomically sharp tip into the sample may destroy the tip. Therefore, the geometry and the materials must be chosen for the body of an STM in such a way that the tip-sample distance does not vary more than a few nanometers during scans. The major constraints are the temperature-dependent changes in STM dimensions and the environmental mechanical vibrations. Since the STMs built for this course were designed to be used under an ambient pressure and temperature, the design choices were different than for STMs designed for ultra high vacuum and extremely low temperatures.

A scanning device consists of a tip, a tip holder, a precision $x-y-z$ actuator and a controller. The tip must be atomically sharp to allow only one path for the tunneling current between the tip and the sample. The thermal expansion of the tip holder should be matched as closely as possible to the rest of the STM body. An actuator must be capable of moving in three-dimensional space with great precision. At this time, piezoelectric actuators are the only ones capable of such precision. There are different types of piezoelectric actuators available that use different controllers. The cost constraint ruled out a number of possible geometric designs for an STM actuator due to the type of controller needed.

The tunneling current between the tip and the sample is extremely low (in the pico-amps range). Its value depends on the voltage between the tip and the sample as well as on the distance between the two. Therefore, by measuring the tunneling current, the distance between the tip and the sample can be inferred. This low tunneling current must be 
amplified and converted to voltage before an analog-to-digital converter (ADC) can measure it. A graduate student designed an amplifier specially for this course.

A controller is used to send the controlling voltages to the $x-y-z$ actuator to perform a scan and thus create an image of the surface just under the tip. To show an image, the image data has to be sent to a display. An adequate display device available in the lab was a PC, so a controller had to be chosen to connect the STM to the PC and transfer the data to it.

\subsubsection{The Low-Cost STM Prototype}

In this laboratory session students were introduced to the current research efforts in creating low-cost STMs. The system's operation was demonstrated after each of its components was discussed in detail. A steady tunneling current was achieved and then an image of the lower aluminum plate of the STM was obtained. Some design decisions in the subsequent student STMs were heavily influenced by this STM solution.

The discussed low-cost STM was originally used in a study of STM vibration isolation systems $^{12}$. The STM was designed to operate under normal ambient conditions. The $x-y-z$ actuator was constructed from a unimorph piezoelectric disk thus allowing application of low controlling voltages. To ensure stable DC voltages and to protect the system from possible electromagnetic interference (EMI) from the power network, batteries were used to generate most of the DC voltages for the STM operation. A unimorph disk consists of two disks bonded together, a metal disk and a piezoelectric disk. When a voltage is applied to the piezoelectric disk, it generates tangential forces and bends the somewhat larger metal disk. The disk's piezoelectric part was cut into four quadrants thus creating an STM X-y-z actuator. To generate motions of the STM tip mounted in the center of the unimorph disk, various voltages (with respect to the metal disk electrode) are applied to the four quadrants of the actuator. Both, x-y scanning and z-motion of the tip are possible when using this actuator.

The tip of the STM was prepared from a platinum/iridium (Pt/Ir) wire. A piece of wire was cut to a length of approximately $1.5 \mathrm{~cm}$. Then, the tip was created by cutting the wire diagonally at an angle of approximately $45^{\circ}$ with a set of sharp wire cutters. Finally, the wire was inserted into a tip holder. The tip holder comprised a plastic tube approximately $1 \mathrm{~cm}$ long having a $5 \mathrm{~mm}$ outer diameter, and a female connector for standard dual-inpack integrated circuits. The inside diameter of the tube allowed the connector to fit securely and accept the Pt/Ir wire tip. In this design, the STM tip can be replaced quickly. Figure 3 depicts one of the unimorph piezoelectric actuators capable of 3-dimensional tip motion that was built during the course of this project. 


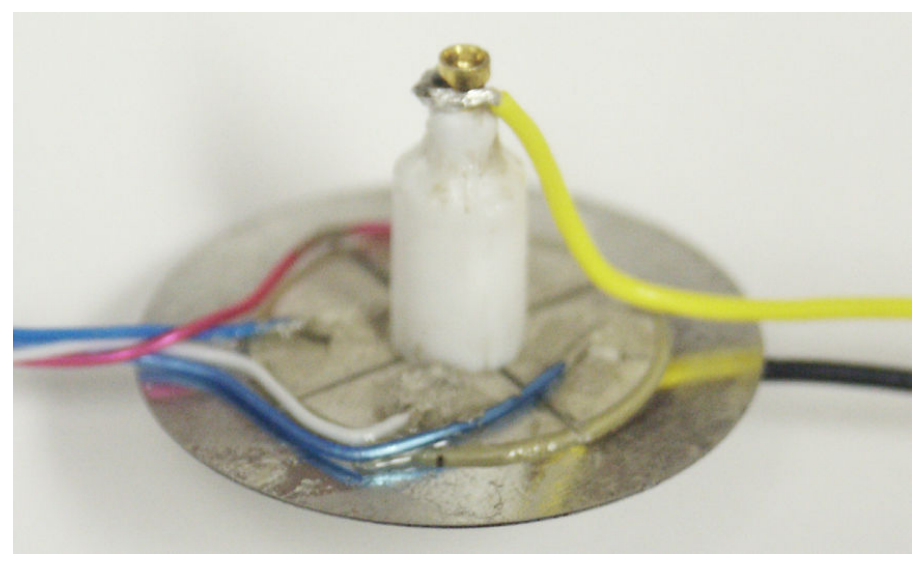

Figure 3. Unimorph piezo-disk actuator

The approach mechanism was not automated. It consisted of two plates and three precision screws ( 80 threads/inch) creating a lever to give a maximum motion reduction. The project calculations showed that for every turn of a screw placed furthest away from the tip, the tip would move $3 \mu \mathrm{m}$. Since the maximum z-motion of the tip created by a voltage $(+/-5 \mathrm{~V})$ applied to the unimorph disk was calculated to be $1.6 \mu \mathrm{m}$, the approach mechanism was appropriate. When the tip was sufficiently close to the surface an approach procedure was implemented to bring the tip into the tunneling current range.

An amplifier was designed and built to amplify the tunneling current and convert it to voltage for use by an analog-to-digital converter (ADC). To control the scan, the voltage waveforms were generated by the computer and sent to a multi-channel digital-to-analog converter (DAC). These functions were performed using a Texas Instruments (TI) microprocessor-based data acquisition and control system, an MSC1211EVM evaluation board. This device consists of a 24-bit ADC with an 8051 microcontroller, 32KB of flash memory, $1 \mathrm{~KB}$ data RAM and four (4) 16-bit DACs.

A vibration isolation system, developed as a part of an MS thesis ${ }^{12}$, was discussed and implemented. The system consisted of two subsystems, a single stage spring suspension subsystem (four bungee cords) and a six stage stacked metal plates and viton rubber subsystem. An $80 \times 45 \times 36 \mathrm{~cm}$ steel metal frame was constructed as a support for the bungee cord suspension subsystem. A suspended Styrofoam box housed the 6-stack portion of the vibration isolation system as well as the STM. The box was covered with aluminum foil for electrical noise isolation. Since microprocessors are strong electric noise generators, the TI board was mounted outside the box.

PC software graphical user interface (GUI) and TI board firmware were designed and implemented. A GUI was developed to allow users to measure the tunneling current, display and store images, change scan parameters and apply filtering functions.

\subsubsection{Material Selection and Design Discussions}

Before constructing their STMs, the students selected appropriate materials and discussed their design ideas. Material selection for the Piezo-disks and the STM body was based on 
a few criteria. The thermal expansion coefficient should be matched to the piezo actuators to prevent large drifts of the tunneling gap width when the instrument's temperature changes (due to drifts in the environmental temperature, or internal heating of the instrument). The base should be mechanically rigid, with high resonance frequency. For the STM body, the materials discussed included aluminum, steel, glass, Plexiglas and Macor (machinable ceramic with thermal expansion coefficient similar to piezomaterials). Glass and Macor were ruled out because glass is not easy to machine and Macor is expensive and not readily available. Aluminum, steel and Plexiglas were used for STM bodies. The bodies themselves were proposed, based on calculations of thermal expansion/retraction of the gap between the top of the STM tip and the sample surface. Vibration problems were addressed by choosing compact designs whenever possible to raise the natural frequency of the STMs.

The mechanical designs revolved around the devices available in the lab. About 30 high precision screws (80 threads per inch) were already purchased for this project, so these screws were incorporated in all designs. For $x-y-z$ actuators, all students modified available unimorph disks. Most of the designs used magnets to secure the unimorph disks to the STM bodies. When proposing coarse approach mechanisms only one group used the manual approach coupled with three high precision screws and a mechanical lever similar to the design depicted in Figure 1. Other designs proposed a rack-and-pinion assembly, a directly driven high precision screw or a lead screw as mechanical motion reduction mechanisms. After some experience with the already existing STM, most of the students decided that an implementation of an automated approach mechanism is necessary. DC motors, servo motors and stepper motors were considered for this task. Since the computer controller was able to supply four outputs with up to 5 volts, a stepper motor with 400 steps/revolution was selected to drive the approach mechanism. Since all student designs proposed stepper motors, a stepper motor driver was built and the firmware was adapted to perform the approach using these motors.

\subsubsection{STM Construction}

In discussions of the preliminary student designs many potential problems and concerns were addressed and the designs changed accordingly. For example, in one design the approach mechanism was changed from manual to automatic after the calculations showed that a device with a manual approach would be hard to construct to achieve the desired resolution and would probably result in a failure. The design choices were not imposed on students. In one design, Plexiglas was used for the body of the STM knowing that this material has a high coefficient of thermal expansion. The material was chosen because it was easy to machine and it was transparent thus giving this STM a modern look.

In another design, the motion reduction was obtained only through the stepper motor and a directly driven precision screw. At 80 threads/inch for the screw and 400 steps/revolution for the stepper motor, the $z$ step resolution of such approach mechanism is $0.8 \mu \mathrm{m}$. This was within the calculated limits of $z$ motion for the unimorph actuator thus allowing the approach algorithm to work correctly. All of the designs used stepper 
motors with unimorph disks. While the computer control of stepper motors was implemented, an actual approach algorithm was not. Therefore, students were manually closing the loop in the coarse approach. They would step the motor and check for tunneling current, repeating the operation until a tunneling current was generated.

A new vibration isolation system was constructed for this project. All STMs used it. It consisted of a single stage spring suspension subsystem (four bungee cords) and a three stage stacked metal plates and rubber subsystem. The vibration system was similar to the one used in the prototype, except that it was more compact, used lead for stacked metal plates and ordinary rubber between the plates. To limit the influence of electromagnetic interference from the environment and the data acquisition board, a Faraday cage was constructed.

\subsubsection{STM Wiring and Testing}

After they were built, all STMs were prepared for testing. Sharp platinum/iridium tips were inserted into appropriate tip holders. For each STM, a stepper motor controller was connected to the data acquisition board for inputs and the stepper motor for outputs. The data acquisition board was connected to a serial port of a PC and then powered. A sample of highly oriented pyrolytic graphite was secured under the STM tip and then connected to a low voltage power source to establish a tunneling current when the tip-sample distance became sufficiently small.

The operation of these lab-built STMs was similar to their commercial counterparts. A sample was secured at the bottom plate of the STM. Electrical conductivity between the sample and the rest of the circuit measuring tunneling current was achieved. The STM was placed into the vibration isolation system and Faraday cage. When the desired tunneling current was established, a scan was performed, and an image obtained and saved. Figures 3 to 5 depict student-designed and built STMs. Figure 7 shows the graphical user interface and a successful image of a feature on the sample. 


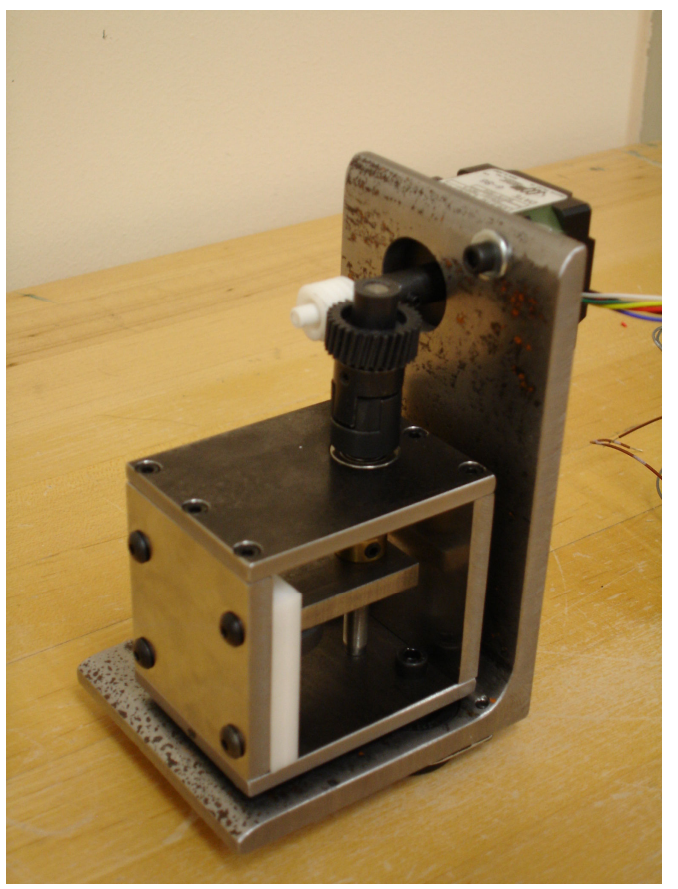

Figure 4. STM using worm-gear

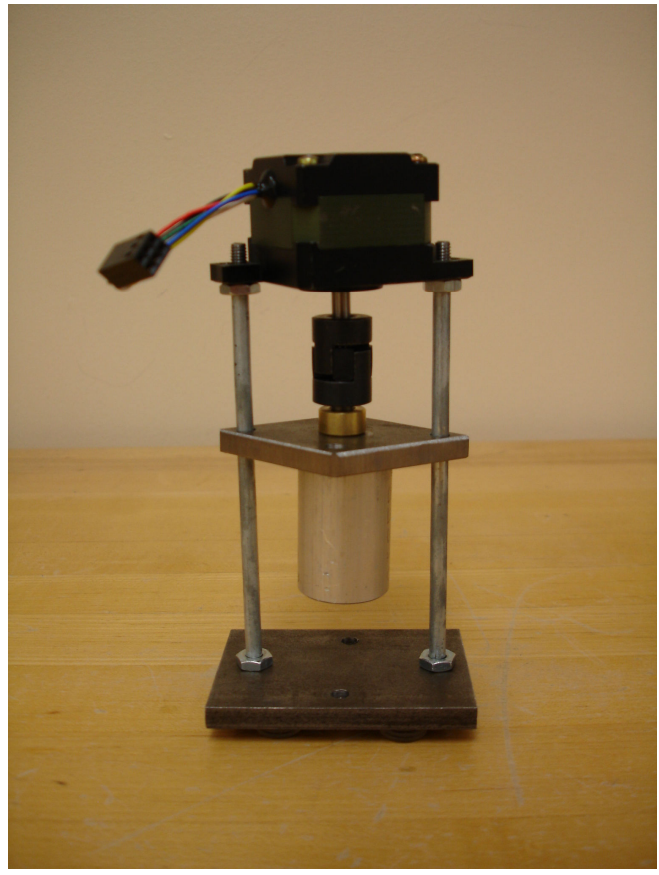

Figure 5. STM using direct drive

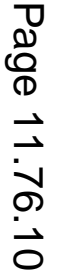




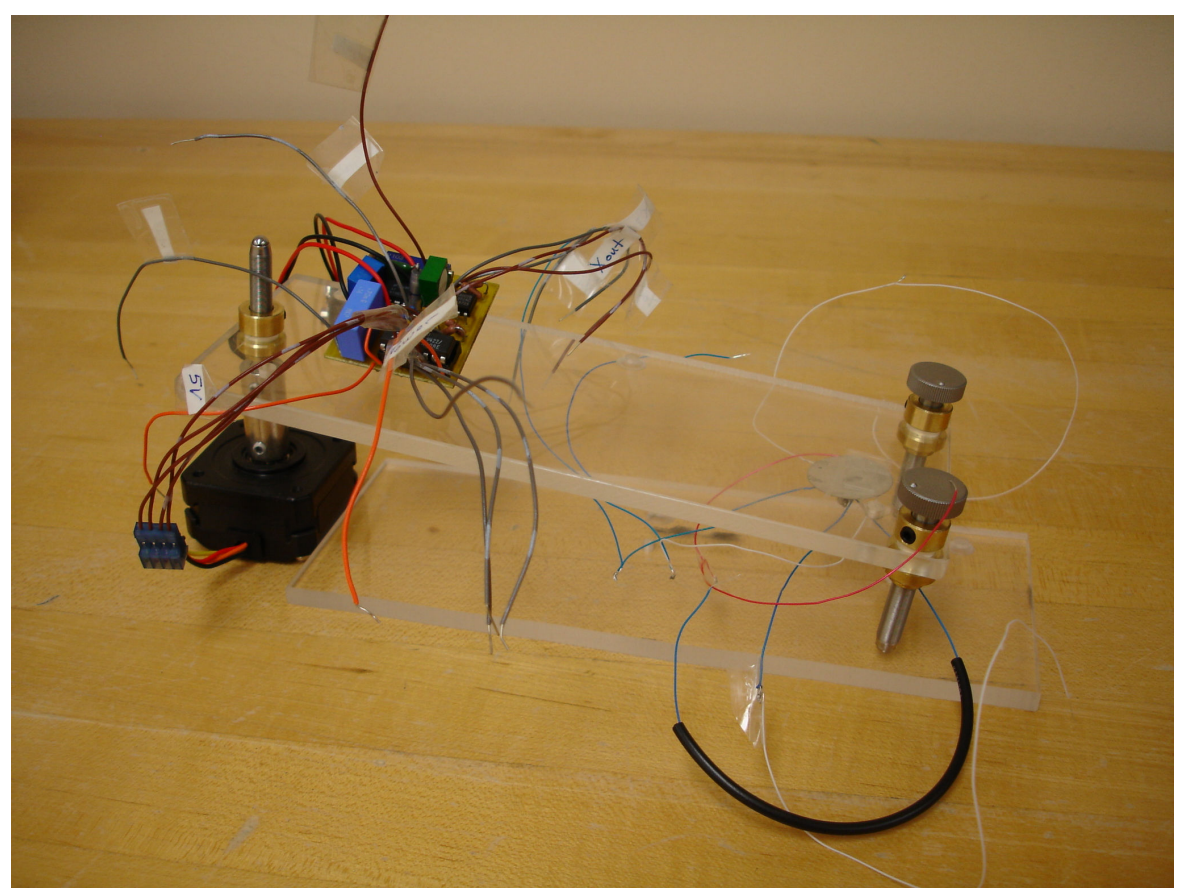

Figure 6. STM with Plexiglas body

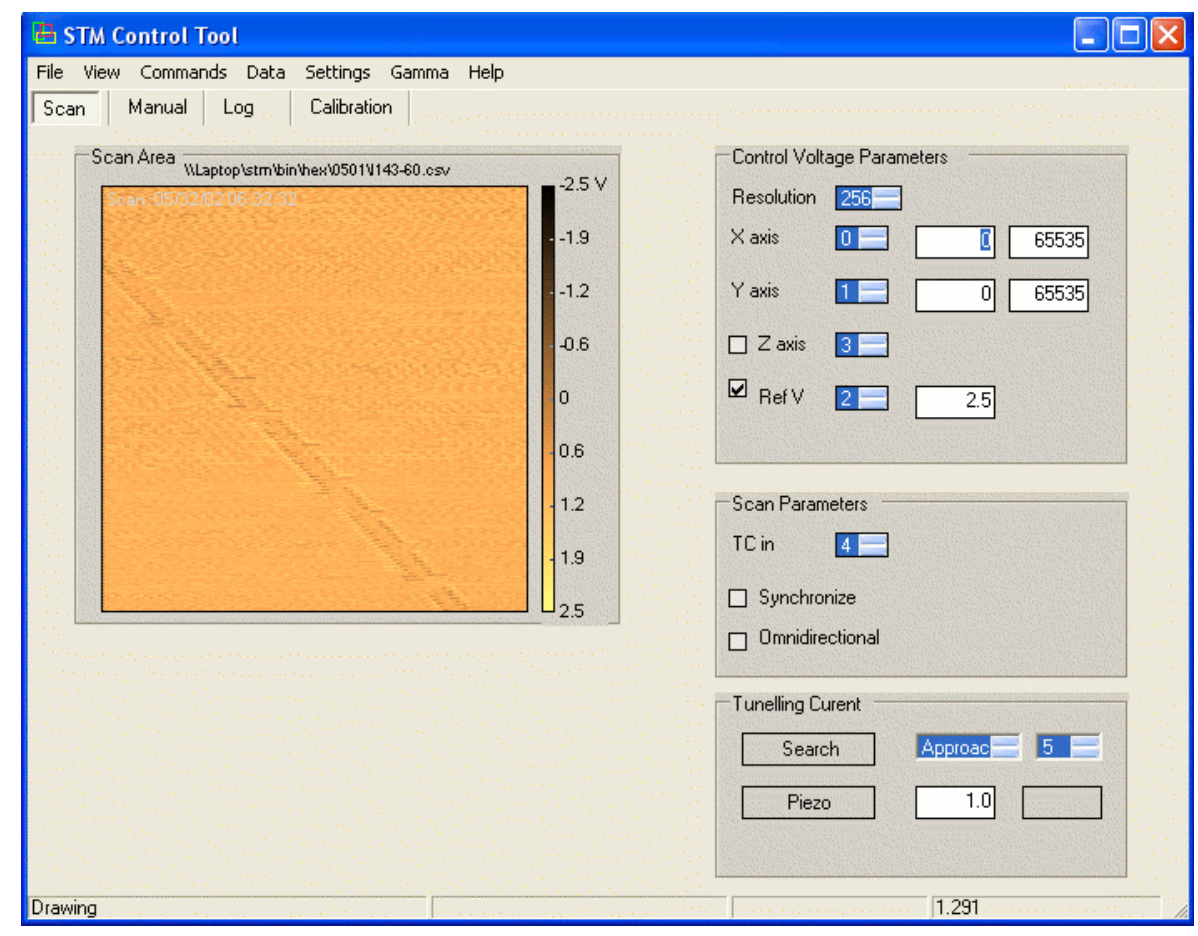

Figure 7. Graphical user interface showing a complete scan.

\subsection{Description of Student-built Scanning Tunneling Microscopes}

All students decided to use stepper motors in their coarse approach mechanisms. All the scanning actuators requiring high-voltages were discarded leaving unimorph piezo-disks 
as scanning devices. These decisions, coupled with $\$ 100$ Texas Instruments high resolution (up to 24-bit acquisition and 16-bit control) data acquisition and control boards, brought the total material cost of student instruments to under $\$ 200$ each, not including manufacturing cost. Table 1 shows a part list with associated costs for one of the STM designs. Two passive vibration isolation systems were designed and implemented. Computer programs providing a graphical user interface, control of the approach mechanism, control of the scanning process and measurement of the tunneling current, as well as an accompanying electric circuit, were developed for this project.

Table 1. Part list for an STM

\begin{tabular}{lc}
\hline Part & Cost (USD) \\
\hline Stepper motor & 5.00 \\
Precision screw & 20.00 \\
Steel cylinder & 5.00 \\
Steel rods & 3.00 \\
Steel Plates & 10.00 \\
Nuts & 1.00 \\
Coupler & 2.00 \\
Magnets & 1.00 \\
Unimorph piezo disk & 2.00 \\
Pt/Ir STM tip & 0.50 \\
Tip holder & 0.50 \\
TI MSC1211EVM evaluation board & 100.00 \\
9V batteries & 4.00 \\
\hline Total: & 154.00 \\
\hline
\end{tabular}

\section{Conclusions}

The effectiveness of the design as a learning tool was assessed through students' performance measurement, informal student interviews and student course evaluations. All students performed well in the laboratory. A summative assessment of the student knowledge gains was obtained through the performance of their STMs. The design objectives for student-built scanning tunneling microscopes were met or exceeded. Each instrument was built well under cost. For each instrument, a sustainable (over 5 minutes) tunneling current was obtained. All design calculations demonstrated the atomic resolution capability of the designs. However, while the scans of highly oriented pyrolytic graphite did show surface characteristics at the lower levels of magnification, the instruments could not distinguish individual atoms.

Every week during the semester students had an informal meeting with the instructor discussing their designs, options and problems. At these times, informal student interviews were performed. Most of the students understood that there is a large difference between a design on paper and a working model. The models proposed were designed on paper and/or on the computer screen. Actual construction of student STMs posed a challenge since it was time intensive. All of the students were satisfied with the 
knowledge acquired. One student termed this "very hard but still the best class I ever had." The students were extremely satisfied when they performed their first successful scans.

At the end of the semester, all students submitted their lab reports describing their STMs and commenting on the laboratory experience in the course. All student comments were positive and encouraging. Student course evaluations were administered at the end of the semester. For this course, the average numerical evaluation was excellent, 4.92 out of 5 .

Acknowledgement: This project was supported in part by NSF Grant 0407289.

\section{References}

[1] Adams, J. D., Rogers, B. S., and Leifer, L. J., "Microtechnology, Nanotechnology, and the ScanningProbe Microscope: An Innovative Course," IEEE Transactions on Education, vol. 47, No. 1, Feb. 2004, pp. 51-56.

[2] McCarthy, B., The 4MAT System: Teaching to Learning Styles With Right/Left Mode Techniques: EXCEL, Inc., 1987.

[3] Kolb, D. A., Experiential Learning: Experience as the Source of Learning and Development, Prentice Hall, 1984.

[4] Harb, J. N., Durant, S. O., and Terry, R. E., "Use of the Kolb learning cycle and the 4MAT system in engineering education," Journal of Engineering Education, vol. 82, No. 2, April 1993, pp. 70-77.

[5] Binnig, G., Rohrer, H., "Scanning Tunneling Microscopy", Helvetica Physica Acta., vol. 55, 1982, pp. 726-735.

[6] Chen, C. J., Introduction to Scanning Tunneling Microscopy, Oxford University Press, 1993.

[7] Wiessendanger, R., (Editor), Scanning Probe Microscopy: Analytical Methods, Springer, 1998.

[8] Marti, O. and Amrein, M., STM and SFM in Biology, Academic Press, 1993.

[9] Alexander, J. D., "Simple STM Project," (Available at: http://www.geocities.com/spm_stm/index.html), 2001.

[10] Muller, J., "STM Project," (Available at: http://www.e-basteln.de), 2001.

[11] Ipek, E., and Maulan, S., "PC-Controlled Scanning Tunneling Microscope," (Available at: http://instruct1.cit.cornell.edu/courses/ee476/FinalProjects/s2002/sm242/index.htm), 2002.

[12] Adali, M., A Vibration Isolation System for a Low-cost Scanning Tunneling Microscope, MS Thesis, 2004.

[13] Dobryanskaya, V., Unimorph Piezo-disk Actuators for a Low-cost Scanning Tunneling Microscope, MS Thesis, 2005.

[14] www.ceeps.colostate-pueblo.edu/nano 